\title{
Polarimetric observations of Hungaria asteroids ${ }^{\star}$
}

\author{
R. Gil-Hutton ${ }^{1}$, D. Lazzaro ${ }^{2}$, and P. Benavidez ${ }^{3}$ \\ ${ }^{1}$ Complejo Astronómico El Leoncito (Casleo) and Universidad Nacional de San Juan, Av. España 1512 sur, J5402DSP, \\ San Juan, Argentina \\ e-mail: rgilhutton@casleo.gov.ar \\ 2 Observatório Nacional, R. Gal. José Cristino 77, 20921-400 Rio de Janeiro, Brazil \\ e-mail: lazzaro@on.br \\ 3 Departamento de Física, Ingeniería de Sistemas y Teoría de la Señal, Universidad de Alicante, Spain
}

Received 26 January 2007 / Accepted 12 March 2007

\section{ABSTRACT}

\begin{abstract}
Aims. We present the results of a polarimetric program at Complejo Astronómico El Leoncito (Casleo), San Juan, Argentina. The aim of this campaign is to estimate the polarimetric properties of asteroids belonging to the Hungaria dynamical group.

Methods. The data were obtained with the Casprof polarimeter at the $2.15 \mathrm{~m}$ telescope. The Casprof polarimeter is a two-hole aperture polarimeter with rapid modulation. The campaign began in 2000, and data on a sample of 24 members of the Hungaria group were obtained. We use the slope - albedo or $P_{\min }$ - albedo relationships to get polarimetric albedos for 18 of these objects.

Results. Only two Xe-type objects, 434 Hungaria and 3447 Burkhalter, shown a polarimetric behavior compatible with a high albedo object. The A-type asteroid 1600 Vyssotsky has a polarimetric behavior similar to what was observed by Fornasier et al. (2006) for 863 Benkolea, and four objects show $P_{\min }$ values consistent with dark surfaces.
\end{abstract}

Key words. solar system: general - techniques: polarimetric - minor planets, asteroids

\section{Introduction}

The Hungaria dynamical group is composed of high-inclination asteroids orbiting at about $1.9 \mathrm{AU}$, just inside the inner edge of the asteroid main belt. They occupy a region with very complex dynamics surrounded by the 5:1 and 4:1 mean motion resonances and the $v_{5}$ and $v_{16}$ secular resonances (Scholl \& Froeschlé 1986). By their location in the inner asteroid belt, the members of this group might be sources of the asteroids that must be replenishing the short-living Mars-crosser population (Michel et al. 2000). The Hungarias are currently clustered in the orbital element space due to long-term dynamical processes, but Williams $(1989,1992)$ and Lemaitre (1994) identified some dynamical clustering in the proper element space, possibly indicating the presence of a family.

To better understand the asteroids in this region of complex dynamics and to aid in assessing of the reality of collisional families, Carvano et al. (2001) performed a spectral survey of asteroid members of the Hungaria group. They observed 29 objects in the visible range and found that 18 of them, presenting a slightly reddish featureless spectrum, could be classified as X-type.

The X-class, originally introduced in the Tholen taxonomy, was defined as spectrally "degenerated", meaning that it is representative of very different mineralogies that could be only recognized by means of the albedo. Three subclasses were thus defined: the E-, M-, and P-types with high, intermediate, and low albedo, respectively (Tholen 1984). The composition associated to each of these sub-classes is very different: carbon/organic-rich silicates for the P-type, metal with traces of silicates or metal

^ Based on observations carried out at the Complejo Astronómico El Leoncito, operated under agreement between the Consejo Nacional de Investigaciones Científicas y Técnicas de la República Argentina and the National Universities of La Plata, Córdoba, and San Juan. plus enstatite for the M-class, and enstatite or other iron-free silicates for the E-type (Gaffey et al. 1989).

In recent years, several works have shed light on diverse characteristics of the X-class spectra: the presence of a $3 \mu \mathrm{m}$ band in the spectra of M- and E-type asteroids (Rivkin et al. 1995, 2000), detection of absorption bands in the visible range on the surface of E-type asteroids (Burbine et al. 1998; Carvano et al. 2001; Fornasier \& Lazzarin 2001), finding absorption bands on M-type asteroids (Busarev 1998; Hardersen et al. 2005), the presence of small absorption bands in the nearinfrared (Clark et al. 2004a), as well as mineralogical differences among E-type asteroids (Clark et al. 2004b). It is noteworthy that the SMASSII spectra revealed variations among the diverse X-class spectra, allowing them to be divided into several sub-classes based solely on spectral features (Bus 1999; Bus \& Binzel 2002).

The location of the Hungaria group in the inner edge of the main belt would then seem to favor a mineralogy associated with E- or M-type classes. This was somehow confirmed by Carvano et al. with the identification of $18 \mathrm{X}$-type asteroids, although they also found eight S-type, two C-type, and one A-type during their spectroscopic survey.

In spite of the above result, which seems to confirm the expected trend, it is important to obtain more information about the physical properties of a statistically significant number of members of the Hungaria group to reach deeper insight into the formation and evolution of these asteroids. In this context, the utmost importance must be given to better knowing their albedos. This kind of information is an essential requirement for any serious attempt at studying the size distribution of the group population and its collisional evolution (Davis et al. 1989; Cellino et al. 1991; Zappalá \& Cellino 1996), but also is a crucial 
parameter for the overall characterization of asteroid surfaces and taxonomic classification (for example, Zellner 1979).

Since the albedo is a valid criterion for sub-classifying $\mathrm{X}$-class objects and one of the most important methods for determining asteroid albedos is polarimetry, an extensive polarimetric observing campaign was developed since 2000 . The aim was to obtain polarimetric observations of Hungaria group members to improve their taxonomic classification and to shed light on their polarimetric properties. Polarimetry is a powerful technique to investigate the physical properties of atmosphereless bodies (see, for example, Muinonen et al. 2002; Shkuratov et al. 2002). For asteroids, it provides two main results: first, the relation between the minimum polarization and the inversion angle of the phase polarization curve is a powerful diagnostic of asteroid surface texture (Dollfus et al. 1977; Dollfus \& Zellner 1979). Using laboratory measurements of meteorites and lunar and terrestrial samples, these authors concluded that asteroids are covered by regolith layers with a broad mixture of particle sizes.

The second important result is an estimation of asteroid albedos using the polarimetric slope - albedo and minimum polarization - albedo relationships (Zellner \& Gradie 1976). The radiation reflected by asteroids is in a general state of partial linear polarization. The observations allow us to determine the so-called Stokes parameters of the incoming light, from which the values for the degree of linear polarization $P$ and the position angle of the polarization plane can be determined at different epochs. The position angle is usually defined relative to the orientation of the scattering plane, which is the plane containing the asteroid, the Sun, and the observer. A classical result of the polarimetric studies of atmosphereless solar system bodies is that the orientation of the plane of linear polarization of the light received from these objects is, as general rule, limited to two cases: it is either parallel or normal to the scattering plane.

In polarimetry, the results of observations are usually expressed using the $P_{r}$ parameter, defined as the ratio:

$P_{r}=\frac{I_{\perp}-I_{\|}}{I_{\perp}+I_{\|}}$

where $I_{\perp}$ and $I_{\|}$are the intensities of the scattered light polarized along the planes perpendicular and parallel to the scattering plane, respectively. It can be shown that $P_{r}=P \cos \left(2 \theta_{r}\right)$, where $\theta_{r}$ is the angle between the polarization angle of the linear polarization plane and the normal to the scattering plane.

In the present paper we show results of the extensive polarimetric observing program of Hungaria asteroids. In Sect. 2 we describe the observations and in Sects. 3 and 4 we present and discuss our results.

\section{Observations}

The observations presented in this paper were carried out during different observing runs between February 2000 and October 2004 at the 2.15-m telescope of Complejo Astronómico El Leoncito (CASLEO), San Juan, Argentina, using the CASPROF polarimeter. CASPROF is a two-hole aperture polarimeter with rapid modulation provided by a rotating achromatic half-wave retarder and Wollaston prism polarizing beamsplitter. The complementary polarized beams are detected with photomultipliers operating in pulse-counting mode, and the acquisition and guiding were accomplished with a CCD camera viewing the sky surrounding the entrance aperture. From the analysis of several standard stars, the instrumental polarization was found fairly constant and always below $0.1 \%$.
The targets were observed during runs some weeks apart to obtain measurements during the same apparition at different phase angles. In order to check the performances of the instrument we observed a few bright asteroids for which polarimetric data were already available in the literature. In these cases, the observations always fit the previously known phase-polarization curves of these objects.

Observing nights were generally assigned around the new Moon to minimize the contamination of sky polarization by moonlight. In all cases, the smallest diaphragm allowed by the observing conditions was used in order to minimize the contribution of sky background, but it was always smaller than $17^{\prime \prime}$. Each night a minimum of two zero-polarization standard stars and one high-polarization one were observed to determine instrumental polarization. The standard stars data were obtained from Turnshek et al. (1990) and Gil-Hutton \& Benavidez (2003).

Due to their faintness, the targets were observed consecutively several times each night with individual exposure times long enough ( $\geq 180 \mathrm{~s}$ ) to reach an acceptable signal. The measurements for each retarder position were co-added to improve the $\mathrm{S} / \mathrm{N}$ ratio, and the errors were evaluated assuming a Poisson distribution. After a correction for instrumental polarization, the Stokes parameters were obtained using reduction programs specially designed for this polarimeter, with some modifications in order to adapt the reduction to the specific needs of asteroid polarimetry, including the computation of the position angle of the scattering plane and the derivation of the $P_{r}$ parameter.

\section{Results}

Data on 24 members of the Hungaria group obtained during different observing runs between February 2000 and October 2004 are presented here. The observed asteroids, date, total integration time in seconds, phase angle, position angle of the scattering plane, degree of linear polarization and its error, position angle in the equatorial reference frame and its error, and $P_{r}$ are shown in Table 1.

Although some targets could be observed at a single phase angle, comparing the data obtained with the polarimetric measurements of asteroids of the same taxonomic type allows interesting conclusions to be drawn about their surface properties. In Table 2 the taxonomic classification of the observed asteroids according to Tholen (1989), Carvano et al. (2001), Bus \& Binzel (2002), and Lazzaro et al. (2004) are listed, and Fig. 1 shows all the polarimetric observations obtained during this campaign for these objects. Since there is only one polarimetric observation in the literature for one member of the Hungaria group (an unpublished observation of 434 Hungaria included in the TRIAD file, Morrison \& Zellner 1979), the observations presented here are the first polarimetric results obtained for members of this dynamical group.

Figure 1 shows that the polarimetric observations of asteroids classified as E-, X-, or Xe-types are mainly in the region of phase angles $\alpha \geq 20^{\circ}$, and they are dispersed in a broad region of phase angle and $P_{r}$. In spite of the common assumption of taxonomic homogeneity for the asteroids belonging to the Hungaria group, this behavior is an indication of the different polarimetric properties of their surfaces.

To find the polarimetric albedo $P_{v}$ for the observed asteroids, two empirical relations linking this parameter with the slope of the phase-polarization curve $h$, calculated around the inversion phase angle $\alpha_{0}$, or the minimum of the phase-polarization 
Table 1. Polarimetric observations of Hungaria asteroids.

\begin{tabular}{|c|c|c|c|c|c|c|c|c|c|}
\hline Asteroid & Date UT & $\begin{array}{c}T_{\text {int }} \\
\text { S }\end{array}$ & $\begin{array}{c}\alpha \\
\alpha \\
\circ\end{array}$ & $\theta_{\odot}$ & $\begin{array}{l}P \\
\%\end{array}$ & $\begin{array}{c}\sigma_{P} \\
\%\end{array}$ & $\begin{array}{l}\theta \\
\circ\end{array}$ & $\sigma_{\theta}$ & $\begin{array}{l}P_{r} \\
\%\end{array}$ \\
\hline \multirow[t]{6}{*}{434 Hungaria } & 2000 Feb. 04 & 2160 & 13.6 & 24.7 & 0.20 & 0.05 & 9.3 & 7.0 & -0.17 \\
\hline & 2001 Jun. 25 & 1440 & 33.8 & 63.1 & 1.00 & 0.03 & 138.1 & 0.9 & 0.87 \\
\hline & 2003 Apr. 01 & 1440 & 9.5 & 128.0 & 0.36 & 0.04 & 122.0 & 3.0 & -0.35 \\
\hline & 2003 May 05 & 1080 & 25.7 & 120.8 & 0.27 & 0.16 & 25.2 & 15.6 & 0.27 \\
\hline & 2004 Aug. 25 & 3600 & 31.4 & 78.5 & 0.60 & 0.05 & 4.6 & 2.4 & 0.51 \\
\hline & 2004 Oct. 14 & 2880 & 21.5 & 110.0 & 0.18 & 0.06 & 33.7 & 9.8 & 0.16 \\
\hline 1019 Strackea & 2004 Aug. 25 & 4320 & 4.6 & 29.3 & 0.57 & 0.08 & 25.4 & 3.9 & -0.57 \\
\hline \multirow[t]{4}{*}{1025 Riema } & 2000 Feb. 03 & 4320 & 13.5 & 157.5 & 0.36 & 0.07 & 172.2 & 5.6 & -0.31 \\
\hline & 2001 Jun. 25 & 4320 & 29.2 & 64.4 & 1.78 & 0.06 & 139.3 & 0.9 & 1.54 \\
\hline & 2001 Jul. 22 & 4320 & 24.7 & 60.8 & 0.99 & 0.15 & 132.4 & 4.3 & 0.79 \\
\hline & 2004 Oct. 15 & 7200 & 15.6 & 158.0 & 0.19 & 0.10 & 153.5 & 13.2 & -0.19 \\
\hline \multirow[t]{3}{*}{1355 Magoeba } & 2003 Apr. 02 & 2880 & 15.1 & 12.6 & 0.45 & 0.11 & 42.2 & 7.1 & -0.23 \\
\hline & 2003 May 05 & 2880 & 20.7 & 94.8 & 0.87 & 0.20 & 137.7 & 6.6 & -0.06 \\
\hline & 2004 Oct. 15 & 3600 & 24.2 & 86.5 & 0.61 & 0.15 & 2.8 & 7.0 & 0.59 \\
\hline \multirow[t]{3}{*}{1453 Fennia } & 2001 Jun. 25 & 4320 & 25.3 & 57.5 & 1.18 & 0.05 & 163.9 & 1.1 & 0.99 \\
\hline & 2001 Jul. 21 & 4320 & 29.4 & 84.5 & 1.69 & 0.04 & 165.0 & 0.7 & 1.60 \\
\hline & 2004 Aug. 26 & 7200 & 16.2 & 98.8 & 1.01 & 0.12 & 64.2 & 3.2 & -0.36 \\
\hline \multirow[t]{4}{*}{1509 Esclangona } & 2003 Jun. 25 & 2880 & 20.3 & 65.4 & 0.25 & 0.10 & 8.6 & 10.9 & 0.10 \\
\hline & 2001 Jul. 21 & 4320 & 9.3 & 13.0 & 0.92 & 0.14 & 7.8 & 4.3 & -0.91 \\
\hline & 2003 Apr. 01 & 2880 & 25.3 & 68.9 & 0.79 & 0.19 & 143.2 & 6.8 & 0.67 \\
\hline & 2003 May 05 & 2880 & 31.6 & 99.0 & 1.53 & 0.24 & 20.3 & 2.7 & 1.41 \\
\hline \multirow[t]{3}{*}{1600 Vyssotsky } & 2000 Nov. 02 & 4320 & 18.3 & 88.4 & 0.77 & 0.10 & 46.4 & 3.7 & -0.08 \\
\hline & 2004 May 18 & 4320 & 25.6 & 128.9 & 0.40 & 0.14 & 30.8 & 12.6 & 0.39 \\
\hline & 2004 Jul. 16 & 4320 & 32.6 & 111.7 & 0.91 & 0.12 & 46.8 & 1.7 & 0.58 \\
\hline 1727 Mette & 2001 Jun. 25 & 2880 & 18.2 & 151.2 & 0.24 & 0.06 & 156.2 & 6.5 & -0.24 \\
\hline 2001 Einstein & 2000 Feb. 03 & 4320 & 27.5 & 106.9 & 1.09 & 0.21 & 3.8 & 5.5 & 0.97 \\
\hline 2131 Mayall & 2000 Feb. 03 & 4320 & 11.8 & 158.1 & 1.04 & 0.15 & 175.3 & 3.7 & -0.86 \\
\hline \multirow{2}{*}{2577 Litva } & 2000 Dec. 01 & 4320 & 28.6 & 145.0 & 0.95 & 0.18 & 55.3 & 5.2 & 0.95 \\
\hline & 2004 May 17 & 4320 & 22.3 & 135.7 & 0.31 & 0.21 & 17.1 & 17.1 & 0.17 \\
\hline 3022 Dobermann & 2004 Aug. 26 & 7200 & 11.6 & 6.5 & 3.40 & 0.37 & 154.2 & 3.1 & -1.46 \\
\hline \multirow[t]{3}{*}{3086 Kalbaugh } & 2000 Feb. 03 & 4320 & 12.5 & 145.5 & 0.65 & 0.11 & 153.6 & 4.7 & -0.62 \\
\hline & 2003 Apr. 01 & 2520 & 23.8 & 150.2 & 0.53 & 0.03 & 33.5 & 1.9 & 0.32 \\
\hline & 2003 May 05 & 2880 & 19.2 & 33.7 & 0.58 & 0.12 & 70.2 & 5.8 & -0.17 \\
\hline 3101 Goldberger & 2000 Feb. 04 & 4320 & 10.0 & 140.3 & 1.09 & 0.21 & 119.2 & 5.5 & -0.81 \\
\hline \multirow[t]{3}{*}{3169 Ostro } & 2000 Nov. 02 & 2160 & 30.3 & 102.6 & 3.36 & 0.31 & 179.5 & 2.6 & 3.02 \\
\hline & 2000 Dec. 01 & 4320 & 19.8 & 107.8 & 0.47 & 0.11 & 156.5 & 6.7 & 0.06 \\
\hline & 2004 Jul. 15 & 4320 & 31.6 & 112.5 & 3.42 & 0.20 & 15.0 & 1.7 & 3.30 \\
\hline \multirow[t]{4}{*}{3447 Burkhalter } & 2000 Feb. 03 & 8640 & 22.5 & 101.4 & 0.10 & 0.08 & 140.2 & 19.4 & -0.02 \\
\hline & 2000 Apr. 14 & 4320 & 19.8 & 110.5 & 0.54 & 0.10 & 98.2 & 5.2 & -0.08 \\
\hline & 2001 Jul. 22 & 4320 & 28.7 & 69.2 & 0.82 & 0.22 & 138.0 & 7.6 & 0.60 \\
\hline & 2003 May 05 & 2880 & 15.0 & 66.7 & 0.12 & 0.08 & 59.4 & 16.0 & -0.12 \\
\hline 4116 Elachi & 2000 Nov. 02 & 2880 & 18.1 & 48.2 & 2.10 & 0.10 & 90.1 & 7.6 & -0.23 \\
\hline 5427 Jensmartin & 2000 Nov. 02 & 4320 & 5.8 & 88.5 & 0.84 & 0.20 & 70.0 & 12.0 & -0.67 \\
\hline $56391989 \mathrm{PE}$ & 2004 Aug. 25 & 6840 & 9.4 & 90.4 & 1.97 & 0.33 & 66.4 & 4.8 & -1.32 \\
\hline 5968 Trauger & 2000 Nov. 02 & 5760 & 13.7 & 153.0 & 1.06 & 0.15 & 158.3 & 4.0 & -1.04 \\
\hline \multirow[t]{3}{*}{6249 Jennifer } & 2000 Nov. 02 & 5760 & 29.5 & 106.9 & 1.14 & 0.18 & 1.2 & 4.6 & 0.98 \\
\hline & 2000 Dec. 01 & 4320 & 22.0 & 144.6 & 0.52 & 0.20 & 108.4 & 10.2 & -0.16 \\
\hline & 2004 May 19 & 6480 & 27.3 & 108.7 & 0.95 & 0.11 & 28.9 & 3.3 & 0.89 \\
\hline \multirow[t]{2}{*}{6911 Nancygreen } & 2000 Nov. 02 & 4320 & 11.6 & 125.7 & 0.84 & 0.16 & 121.7 & 5.5 & -0.83 \\
\hline & 2000 Dec. 01 & 4320 & 18.1 & 32.2 & 0.38 & 0.09 & 175.9 & 6.2 & -0.11 \\
\hline \multirow[t]{2}{*}{7187 Isobe } & 2001 Jul. 22 & 2880 & 10.1 & 25.0 & 1.45 & 0.27 & 13.9 & 10.8 & -1.34 \\
\hline & 2003 Apr. 02 & 2880 & 19.1 & 81.8 & 0.08 & 0.10 & 21.3 & 12.9 & 0.04 \\
\hline \multirow[t]{2}{*}{$135781993 \mathrm{MK}$} & 2001 Jun. 25 & 4320 & 9.6 & 64.3 & 1.36 & 0.16 & 148.6 & 3.4 & -0.75 \\
\hline & 2001 Jul. 21 & 4320 & 17.9 & 105.1 & 0.05 & 0.06 & 121.8 & 23.9 & -0.04 \\
\hline
\end{tabular}

curve $P_{\min }$ were used. These relations are expressed by means of very simple mathematical forms:

$\log p_{v}=C_{1} \log h+C_{2}$

$\log p_{v}=C_{3} \log P_{\min }+C_{4}$

where $C_{1}, C_{2}, C_{3}$, and $C_{4}$ are constants. In this paper we use the set of constants proposed by Cellino et al. (1999), namely: $C_{1}=-1.118 \pm 0.071, C_{2}=-1.779 \pm 0.062, C_{3}=-1.357 \pm$ 0.140 , and $C_{4}=-0.858 \pm 0.030$.
The adopted criterion for the determination of the polarimetric slope $h$ was that this parameter can be obtained from a linear least-square fit, only when at least three measurements performed in different nights are available. The linear least-square fits were obtained after assigning a weight to each measurement, inversely proportional to its nominal uncertainty derived from the data reduction procedure. Since we did not actually have the possibility of sampling the polarization curve in great detail around the phase angle where the maximum of the negative 
Table 2. Taxonomic classification of Hungaria asteroids.

\begin{tabular}{lllll}
\hline \hline Asteroid & Class $^{a}$ & Class $^{b}$ & Class $^{c}$ & Class $^{d}$ \\
\hline 434 Hungaria & $\mathrm{E}$ & $\mathrm{Xe}$ & $\mathrm{Xe}$ & $\mathrm{X} / \mathrm{Xe}$ \\
1019 Strackea & $\mathrm{S}$ & & & \\
1025 Riema & $\mathrm{E}$ & $\mathrm{Xe}$ & $\mathrm{Xe}$ & $\mathrm{X} / \mathrm{Xe}$ \\
1355 Magoeba & $\mathrm{X}$ & $\mathrm{Xe}$ & $\mathrm{X} / \mathrm{Xe}$ & \\
1453 Fennia & $\mathrm{S}$ & & & \\
1509 Esclangona & $\mathrm{S}$ & $\mathrm{S}$ & $\mathrm{A} / \mathrm{Ld}$ & \\
1600 Vyssotsky & & $\mathrm{A}$ & $\mathrm{A}$ & $\mathrm{A} / \mathrm{A}$ \\
1727 Mette & $\mathrm{S}$ & & & \\
2001 Einstein & $\mathrm{X}$ & $\mathrm{Xe}$ & $\mathrm{Xe}$ & $\mathrm{X} / \mathrm{Xe}$ \\
2131 Mayall & $\mathrm{S}$ & & $\mathrm{S}$ & \\
2577 Litva & $\mathrm{EU}$ & & & $\mathrm{S} / \mathrm{Sl}$ \\
3022 Dobermann & & & & $\mathrm{X} / \mathrm{Xk}$ \\
3086 Kalbaugh & & & & \\
3101 Goldberger & & $\mathrm{Xe}$ & & $\mathrm{X} / \mathrm{Xe}$ \\
3169 Ostro & $\mathrm{TS}$ & $\mathrm{C}$ & $\mathrm{Xe}$ & $\mathrm{C} / \mathrm{Cb}$ \\
3447 Burkhalter & & $\mathrm{Xe}$ & & $\mathrm{X} / \mathrm{Xe}$ \\
4116 Elachi & & $\mathrm{S}$ & $\mathrm{Sl}$ & $\mathrm{S} / \mathrm{Sl}$ \\
5427 Jensmartin & & & & \\
5639 1989 PE & & $\mathrm{C}$ & & $\mathrm{C} / \mathrm{B}$ \\
5968 Trauger & & & & \\
6249 Jennifer & & $\mathrm{Xe}$ & & \\
6911 Nancygreen & & & & \\
7187 Isobe & & & & \\
13578 1993 MK & & & & \\
\hline
\end{tabular}

${ }^{a}$ Tholen (1989); ${ }^{b}$ Carvano et al. (2001); ${ }^{c}$ Bus \& Binzel (2002);

${ }^{d}$ Lazzaro et al. (2004).

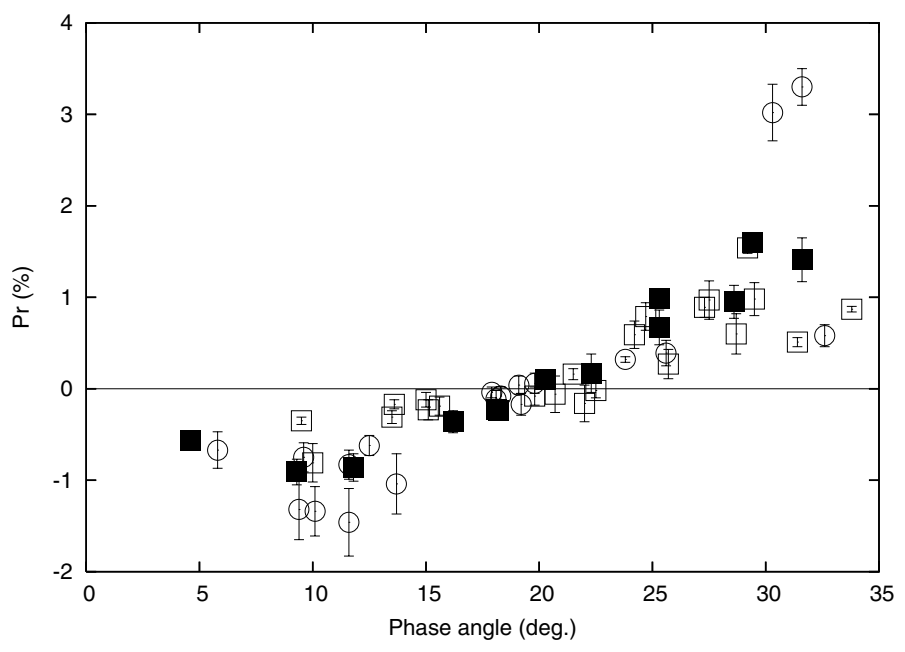

Fig. 1. Polarimetric observations for Hungaria group members. Data for E-, X-, and Xe-type objects are indicated by squares, for S-type objects by filled squares, and other objects by circles.

polarization branch is found $\left(\alpha_{\min }\right)$, we decided to rely on more general results available in the literature, and we accepted only measurements obtained in an interval of two degrees of phase centered around the value of $10^{\circ}$ as reasonable determinations for $P_{\min }$. We are aware that this procedure may be not completely satisfactory, as it contains some elements of subjectivity. We are also aware that in any case the $P_{\min }$ - albedo relationship is not as strict as the slope - albedo one.

For each observed asteroid, the polarimetric parameters $P_{\min }$, $\alpha_{\min }, h, \alpha_{0}$, and the polarimetric albedo $p_{v}$ obtained are listed in Table 3. We obtained polarimetric albedos for eleven asteroids using the slope - albedo relationship and for nine objects using the $P_{\text {min }}$ - albedo relationship. For two asteroids, 434 Hungaria and 1509 Esclangona, the polarimetric albedos were obtained using both relationships.

Four of our targets have albedos obtained using different methods. Morrison \& Zellner (1979) listed an albedo of $p_{v}=$ 0.428 for 434 Hungaria in the TRIAD data compilation, but this value is based only on an unpublished result of $P_{\min }=0.32$. The asteroids 1453 Fennia, 1509 Esclangona, and 2131 Mayall were observed by IRAS for the IRAS Minor Planet Survey (Tedesco et al. 1992, 2002), resulting in radiometric albedos of $0.281 \pm$ $0.035,0.232 \pm 0.038$, and $0.239 \pm 0.031$, respectively. These values are higher than those presented here, but for small objects the IRAS Minor Planet Survey radiometric albedos show significant discrepancies with results obtained by radar (Pettengill \& Jurgens 1979) and polarimetry (Cellino et al. 1999, 2005), in the sense that the radiometric albedos are significantly greater. This difference is due to the assumption made in the reduction process about the distribution of temperature on the asteroid surface and also in the value of some constants present in the relation describing the equilibrium between the absorbed and emitted radiation on an asteroid surface (Lebofsky et al. 1986; Lebofsky \& Spencer 1989).

In the observed sample of E-, X-, or Xe-type objects, only the asteroids 434 Hungaria and 3447 Burkhalter show the high polarimetric albedos $\left(p_{v} \geq 0.3\right)$ characteristic of E-type objects. On the other hand, the asteroids 1025 Riema, 1355 Magoeba, 3101 Goldberger, and 6249 Jennifer have polarimetric albedos in the range observed for M-type asteroids, $0.13 \leq p_{v} \leq 0.22$, with values for $P_{\min }, \alpha_{\min }$, and $\alpha_{0}$, which is also consistent with those observed for objects with this taxonomic type (Gil-Hutton 2007). The S-type asteroids 1453 Fennia, 1509 Esclangona, 2131 Mayall, and 2577 Litvia show polarimetric albedos that are consistent with their taxonomic type.

The A-type asteroid 1600 Vyssotsky shows the polarimetric behavior typical of high albedo objects. The polarimetric slope for this asteroid is similar to what has been obtained by Fornasier et al. (2006) for the A-type object 863 Benkoela. The asteroid 3169 Ostro is a special case: it was classified as TS, C, and Xe by different authors (see Table 2), but the polarimetric albedo reported here shows that it is a dark object consistent with a C-type surface as 56391989 PE. Finally, the asteroids 3022 Dobermann and 7187 Isobe show $P_{\text {min }}$ values that are consistent with dark surfaces. The former, due to its X-type spectrum, can be classified as P-type, while the latter can be either a C- or P-type.

\section{Discussion}

In the present work we have presented polarimetric observations of 24 members of the Hungaria group. As a general result, we obtained polarimetric albedos for 18 objects using the slope albedo or $P_{\min }$ - albedo relationships.

Spectroscopically, the Hungaria group appears to be dominated by E-, X-, or Xe-type objects (Carvano et al. 2001), but only two of the observed asteroids in these classes, 434 Hungaria and 3447 Burkhalter, show a polarimetric behavior compatible with a high albedo object. The four other observed asteroids with these taxonomic classes (1025 Riema, 1355 Magoeba, 3101 Goldberger, and 6249 Jennifer) have polarimetric albedos in the range observed for M-type asteroids.

The A-type asteroid 1600 Vyssotsky shows a polarimetric behavior similar to what was observed by Fornasier et al. (2006) for 863 Benkolea, also an A-type. Both objects show polarimetric slopes characteristic of high albedo asteroids.

Four objects show $P_{\min }$ values consistent with dark surfaces: 3022 Dobermann, 3169 Ostro, 56391989 PE, and 7187 Isobe. 
Table 3. Estimated polarimetric parameters for Hungaria asteroids.

\begin{tabular}{lccccc}
\hline \hline Asteroid & $\begin{array}{c}\left|P_{\min }\right| \\
\%\end{array}$ & $\begin{array}{c}\alpha_{\min } \\
{ }^{\circ}\end{array}$ & $\begin{array}{c}h \\
\% /^{\circ}\end{array}$ & $\begin{array}{c}\alpha_{0} \\
{ }^{\circ}\end{array}$ & albedo \\
\hline 434 Hungaria & & & $0.050 \pm 0.003$ & 17.7 & $0.470 \pm 0.125$ \\
& $0.35 \pm 0.04$ & 9.5 & & & $0.576 \pm 0.129$ \\
1025 Riema & & & $0.128 \pm 0.009$ & 17.3 & $0.166 \pm 0.036$ \\
1355 Magoeba & & & $0.083 \pm 0.020$ & 18.2 & $0.267 \pm 0.095$ \\
1453 Fennia & & & $0.149 \pm 0.009$ & 18.6 & $0.140 \pm 0.029$ \\
1509 Esclangona & & & $0.116 \pm 0.022$ & 19.4 & $0.185 \pm 0.055$ \\
& $0.91 \pm 0.14$ & 9.3 & & & $0.158 \pm 0.035$ \\
1600 Vyssotsky & & & $0.047 \pm 0.011$ & 19.5 & $0.506 \pm 0.187$ \\
2131 Mayall & $0.86 \pm 0.15$ & 11.8 & & & $0.170 \pm 0.042$ \\
2577 Litva & & & $0.124 \pm 0.044$ & 20.9 & $0.172 \pm 0.077$ \\
3022 Dobermann & $1.46 \pm 0.37$ & 11.6 & & & $0.083 \pm 0.029$ \\
3086 Kalbaugh & & & $0.107 \pm 0.027$ & 20.8 & $0.202 \pm 0.072$ \\
3101 Goldberger & $0.81 \pm 0.21$ & 10.0 & & & $0.185 \pm 0.066$ \\
3169 Ostro & & & $0.276 \pm 0.018$ & 19.6 & $0.070 \pm 0.013$ \\
3447 Burkhalter & & & $0.068 \pm 0.026$ & 21.9 & $0.336 \pm 0.164$ \\
5639 1989 PE & $1.32 \pm 0.33$ & 9.4 & & & $0.095 \pm 0.033$ \\
6249 Jennifer & & & $0.164 \pm 0.035$ & 22.4 & $0.126 \pm 0.038$ \\
6911 Nancygreen & $0.83 \pm 0.16$ & 11.6 & & & $0.179 \pm 0.049$ \\
7187 Isobe & $1.34 \pm 0.27$ & 10.1 & & & $0.093 \pm 0.027$ \\
13578 1993 MK & $0.75 \pm 0.16$ & 9.6 & & & $0.205 \pm 0.062$ \\
\hline
\end{tabular}

The polarimetric behaviour of 3169 Ostro confirms the C-type classification proposed by Carvano et al. (2001) and Lazzaro et al. (2004).

The results here challenge the current view of the Hungaria group as mainly formed by asteroids with a high albedo and probably linked to the enstatite achondrite meteorites (Zellener et al. 1977; Tholen 1984; Bell et al. 1989; Carvano et al. 2001; Fornasier \& Lazzarin 2001; Clark et al. 2004b). According to the extensive survey of the Hungaria group performed by Carvano et al. (2001) among the 29 asteroids studied, 18 were classified as Xe-, 8 as S-, 2 as C-, and 1 as A-type. We recall that the Xe class has been introduced in the Bus taxonomy (Bus \& Binzel 2002) and it has been generally linked to the E class of the Tholen taxonomy (Tholen 1984). Although both taxonomies start from a slightly reddish spectra, the Tholen one uses the albedo to distinguish the E class among the members of a "degenerate" X class, while the Bus one identifies a small absorption band, at about $0.49 \mu \mathrm{m}$, in an Xe-type object. It is important to note that there is not a perfect correlation among the objects classified in both taxonomies, even if many of the asteroids classified by Tholen (1989) as E also have a Xe classification in the SMASS and S3OS2 surveys (Bus \& Binzel 2002; Lazzaro et al. 2004).

The most representative of asteroids with both $\mathrm{E}$ and Xe taxonomic classifications are 434 Hungaria, 44 Nysa, and 64 Angelina. Recently, Clark et al. (2004b) performed the first detailed compositional modeling of a sample of nine E-type asteroids. Their main result was that the E-type can be separated into three groups according to their inferred composition: "Nysalike", with silicate mineralogy higher in iron than the mineral estatite; "Angelina-like", consistent with silicate mineralogy; and "Hungaria-like" which is not inconsistent with aubrites. Among the nine asteroids of their sample, three are members of the Hungaria group classified as E type by Tholen $(1989)^{1}$.

${ }^{1}$ It is noteworthy that an IRAS albedo is only know for 434 Hungaria and 1102 Sequoia, 0.46 and 0.48 , respectively. According to the authors the third one, 2048 Dwornik, was assigned to the E class based on unpublished albedo provided by J. Gadie and E. F. Tedesco to D. Tholen (Clark et al. 2004b).
However, their mineralogical analysis indicates that two of these objects, 434 Hungaria and 2048 Dwornik, are similar and could be assigned to the "Hungaria-like" group while the third one, 1103 Sequoia, has a composition that is more similar to objects of the "Nysa-like".

Clark et al. (2004b) gives us the second hint that not all the members of the Hungaria group have a similar mineralogy. The first hint has already been given by Carvano et al. (2001) discovering that among the studied 29 asteroids members of the group, as much as 11 of them did not present a spectrum compatible with an Xe-type classification. The present work further strengthens this new scenario for the Hungaria group. In particular, we derived the albedo for 7 asteroids among the 18 Xe classified by Carvano et al. (2001), thus allowing the following refined Tholen taxonomic classification: 4 M-, 2 E-, and 1 P-type. This gives roughly a percentage of $60 \%, 30 \%$, and $15 \%$, respectively, which would give a final distribution of $10 \mathrm{M}-, 5 \mathrm{E}-$, and 3 P-type when extrapolated to all the sample of 18 Xe-type asteroids. Obviously this is a very gross estimate, but it shows that most of the objects in the Hungaria group probably do not have a high albedo.

The results presented here imply that the compositional distribution in the Hungaria group is not as peculiar as presently assumed, with the number of high albedo asteroids in the group compatible to that of the inner main belt. Although quite different techniques and analyses seem to lead to the above conclusion, it is important to stress that they are based on a small sample of objects. Therefore, only more data, both spectroscopic and polarimetric, will allow better constraints on the physical properties and, consequently, a better understanding of the formation and evolution of the Hungaria group of asteroids.

Acknowledgements. D.L. has been supported by CNPq and FAPERJ through diverse fellowships and grants.

\section{References}

Bell, J. F., Davis, D., Hartmann, W., \& Gaffey, M. 1989, in Asteroids II, ed. R. P. Binzel, T. Gehrels, \& M. S. Matthews (Tucson: Univ. of Arizona Press), 921 Burbine, T. H., Cloutis, E. A., Bus, S. J., Meibom, A., \& Binzel, R. P. 1998, BAAS, 30, 1025 
Bus, S. J. 1999, Compositional structure in the asteroid belt: Results of a spectroscopic survey, Ph.D. Thesis, Massachusetts Institute of Technology, Cambridge

Bus, S. J., \& Binzel, R. P. 2002, Icarus, 158, 146

Busarev, V. V. 1998, Icarus, 131, 32

Carvano, J. M., Lazzaro, D., Mothé-Diniz, T., \& Angeli, C. 2001, Icarus, 149, 173

Cellino, A., Zappalá, V., \& Farinella, P. 1991, MNRAS, 253, 561

Cellino, A., Gil-Hutton, R., Tedesco, E. F., Di Martino, M., \& Brunini, A. 1999 Icarus, 138,129

Cellino, A., Gil-Hutton, R., Di Martino, M., et al. 2005, Icarus, 179, 304

Clark, B. E., Bus, S. J., Rivkin, A. S., Shepard, M. K., \& Shah, S. 2004a, AJ, 128,3070

Clark, B. E., Bus, S. J., Rivkin, A. S., et al. 2004b, J. Geophys. Res., 109, E02001

Davis, D. R., Weidenschilling, S. J., Farinella, P., Paolicchi, P., \& Binzel, R. P. 1989, in Asteroids II, ed. R. P. Binzel, T. Gehrels, \& M. S. Matthews (Tucson: Univ, of Arizona Press), 805

Dollfus, A., \& Zellner, B. 1979, in Asteroids, ed. T. Gehrels (Tucson: Univ. of Arizona Press), 170

Dollfus, A., Geake, J. E., Mandevill, J. C., \& Zellner, B. 1977, in Asteroids, Comets, Meteors, ed. A. H. Delsemme (Toledo: Univ. of Toledo Press), 243

Fornasier, S., \& Lazzarin, M. 2001, Icarus, 152, 127

Fornasier, S., Belskaya, I. N., Shkuratov, Yu. G., et al. 2006, A\&A, 455, 371

Gaffey, M. J., Bell, J. F., \& Cruikahnk, D. P. 1989, in Asteroids II, ed. R. P. Binzel, T. Gehrels, \& M. S. Matthews (Tucson: Univ. of Arizona Press), 98

Gil-Hutton, R. 2007, A\&A, 464, 1127

Gil-Hutton, R., \& Benavidez, P. 2003, MNRAS, 345, 97

Hardersen, P. S., Gaffey, M. J., \& Abell, P. A. 2005, Icarus, 175, 141

Lazzaro, D., Angeli, C. A., Carvano, J. M., et al. 2004, Icarus, 172, 179

Lebofsky, L. A., \& Spencer, J. R. 1989, in Asteroids II, ed. R. P. Binzel, T. Gehrels, \& M. S. Matthews (Tucson: Univ. of Arizona Press), 128

Lebofsky, L. A., Sykes, M. V., Tedesco, E. F., et al. 1986, Icarus, 68, 239

Lemaitre, A. 1994, in Seventy-five Years of Hirayama Asteroid Families: The Role of Collisions in the Solar System History, ed. Y. Kozai, R. P. Binzel, \& T. Hirayama (San Francisco: ASP), Conf. Ser., 63, 140

Lemaitre, A., \& Morbidelli, A. 1994, Celest. Mech., 60, 29
Lupishko, D. F., \& Mohamed, R. A. 1996, Icarus, 119, 209

Michel, P., Migliorini, F., Morbidelli, A., \& Zappalà, V. 2000, Icarus, 145, 332

Morrison, D., \& Zellner, B. 1979, in Asteroids, ed. T. Gehrels (Tucson: Univ. of Arizona Press), 1090

Muinonen, K., Piironen, J., Shkuratov, Yu. G., Ovcharenko, A. A., \& Clark, B. E. 2002, in Asteroids III, ed. W. Bottke, R. Binzel, A. Cellino, \& P. Paolicchi (Tucson: University of Arizona Press), 123

Pettengill, G. H., \& Jurgens, R. F. 1979, in Asteroids, ed. T. Gehrels (Tucson: Univ. of Arizona Press), 206

Rivkin, A. S., Howell, E. S., Britt, D. T., et al. 1995, Icarus, 117, 90

Rivkin, A. S., Howell, E. S., Lebofsky, L. A., Clark, B. E., \& Britt, D. T. 2000, Icarus, 145,351

Scholl, H., \& Froeschlé, Ch. 1986, A\&A, 170, 138

Shkuratov, Yu., Ovcharenko, A., Zubko, E., et al. 2002, Icarus, 159, 396

Tedesco, E., Veeder, G., Fowler, J., \& Chillemi, J. 1992, The IRAS Minor Planet Survey, Phillips Laboratory Technical Report PL-TR-92-2049, Hanscom Air Force Base, MA

Tedesco, E. F., Noah, P. V., Noah, M., \& Price, S. D. 2002, AJ, 123, 1056

Tholen, D. J. 1984, Asteroid taxonomy from cluster analysis of photometry, PhD. dissertation (Tucson: Univ. of Arizona)

Tholen, D. J. 1989, in Asteroids II, ed. R. P. Binzel, T. Gehrels, \& M. S. Matthews (Tucson: Univ. of Arizona Press), 1139

Turnshek, D. A., Bohlin, R. C., Williamson II, R. L., Lupie, O. L., \& Koornneef, J. 1990, AJ, 99, 1243-1261, 1344-1377

Williams, J. G. 1989, in Asteroids II, ed. R. P. Binzel, T. Gehrels, \& M. S. Matthews (Tucson: Univ. of Arizona Press), 1034

Williams, J. G. 1992, Icarus, 96, 251

Zappalá, V., \& Cellino, A. 1996, in Completing the Inventory of the Solar System, ed. T. W. Rettig, \& J. H. Hahn (San Francisco: ASP), Conf. Ser., 107,29

Zellner, B. 1979, in Asteroids, ed. T. Gehrels (Tucson: Univ. of Arizona Press), 783

Zellner, B., \& Gradie, J. 1976, AJ, 81, 262

Zellner, B., Leake, M., Morrison, D., \& Williams, J. G. 1977, Geochim. Cosmochim. Acta, 41, 1759 